\title{
Choque séptico por tuberculosis diseminada con absceso esplénico tuberculoso
}

\author{
Septic shock from disseminated tuberculosis with splenic tuberculous abscess
}

\begin{abstract}
Hernando A. Vázquez-Sánchez ${ }^{* 1}$, Fabián González-Loranca², Emmanuel G. Jiménez-Villanueva³, Osmar A. Marroquín-García y Daniela Castro-Martínez ${ }^{4}$

${ }^{1}$ Departamento de Cirugía General, Hospital Regional de Alta Especialidad Ciudad Salud, Tapachula, Puerto Madero, Chiapas; ${ }^{2}$ Departamento de Medicina Interna, Hospital General de México Dr. Eduardo Liceaga, Ciudad de México; ${ }^{3}$ Departamento de Infectología, Hospital Regional de Alta Especialidad Ciudad Salud, Tapachula, Puerto Madero, Chiapas; ${ }^{4}$ Departamento de Medicina Interna, Hospital Juárez de México, Ciudad de México. México
\end{abstract}

\section{Resumen}

La tuberculosis esplénica es poco conocida y de difícil diagnóstico por su escasa presentación de síntomas. Corresponde a una de las formas de presentación extrapulmonar de la enfermedad, las cuales tienen mayor asociación con estados de inmunocompromiso, como la diabetes mellitus. Presentamos el caso de un paciente con diabetes mellitus que ingresó con choque séptico secundario a un probable absceso esplénico, requiriendo intervención quirúrgica en la que se llevó a cabo esplenectomía total y estudio histopatológico, con identificación de Mycobacterium tuberculosis en la pieza quirúrgica.

Palabras clave: Tuberculosis esplénica. Absceso. Cirugía.

\begin{abstract}
Splenic tuberculosis is a little-known and difficult to diagnose entity due to its low presentation of symptoms. It corresponds to one of the forms of extrapulmonary presentation of the disease, which in turn have a greater association with immunocompromised states such as diabetes mellitus. We present the case of a patient with diabetes mellitus who was admitted in the context of septic shock secondary to a probable splenic abscess, requiring surgical intervention. A total splenectomy was performed, managing to isolate Mycobacterium tuberculosis from the surgical specimen.
\end{abstract}

Key words: Splenic tuberculosis. Abscess. Surgery.

\section{Introducción}

La tuberculosis es una enfermedad infecciosa granulomatosa causada por Mycobacterium tuberculosis, que se presenta con una amplia gama de manifestaciones clínicas. Tiene un comportamiento crónico en la mayoría de los casos, aunque a veces se manifiesta de forma aguda y agresiva. Dentro de las formas extrapulmonares, la localización esplénica es de las menos frecuentes y puede confundirse con otras patologías,

\section{Correspondencia:}

*Hernando A. Vázquez-Sánchez

Carretera Tapachula Puerto Madero s/n, km. $15+200$

Carretera Federal 225, Col. Los Toros

Fecha de recepción: 25-09-2020

C.P. 30830, Tapachula, Puerto Madero, Chiapas

E-mail: vasanheral@ @otmail.com
Cir Cir. 2021;89(S1):33-36

Contents available at PubMed www.cirugiaycirujanos.com 
como neoplasias, abscesos piógenos o enfermedades infiltrativas. Para realizar el diagnostico se requieren estudios de imagen, así como estudio histopatológico para su conformación.

Presentamos el caso de un paciente de 80 años que ingresa por fiebre recurrente e inestabilidad hemodinámica, en quien tras descartar otras infecciones y observarse en estudio de imagen la presencia de un probable absceso esplénico se procede a esplenectomía total con estudio de pieza quirúrgica por reacción en cadena de la polimerasa (PCR) y biopsia, confirmando la infección por $M$. tuberculosis.

\section{Caso clínico}

Varón de 80 años, originario de Tapachula, Chiapas, campesino, con diagnóstico de diabetes mellitus tipo 2 de larga evolución. Antecedente de abdominoplastia inguinal en enero de 2020 con colocación de malla. Inicia su padecimiento actual con un cuadro clínico de aproximadamente 2 meses de evolución caracterizado por fiebre recurrente acompañada de escalofríos y cambios en el patrón intestinal, recibiendo múltiples esquemas antibióticos por sospecha de gastroenteritis bacteriana, con escasa mejoría clínica. Tras la exacerbación de la fiebre acude a una institución, donde por sospecha de infección urinaria complicada se le realiza estudio de imagen que solo evidencia crecimiento prostático, por lo que se lleva a cabo resección transuretral de próstata el 2 de marzo de 2020, sin eventualidades. El estudio histopatológico concluye crecimiento prostático benigno sin datos de malignidad. Tras recibir antibióticos, sin mejoría clínica y con recurrencia de la fiebre, es referido a nuestro hospital el 18 de marzo de 2020 para continuar su estudio. A su ingreso se encuentra hemodinámicamente estable, somnoliento, con dolor abdominal en marco cólico y matidez en el espacio semilunar de Traube. En el estudio bioquímico llamaba la atención la presencia de trombocitopenia, leucocitos con rangos normales, hiperlactatemia, hipoalbuminemia y patrón colestásico. El examen general de orina revela presencia de piocitos, eritrocitos y bacterias abundantes, nitritos negativos, y escasas levaduras.

Debido a que el paciente continuó con deterioro clínico, se decidió solicitar estudios de gabinete (Fig. 1). Por ultrasonido se detectó una colección localizada en el bazo, y la tomografía computada mostró el parénquima pulmonar con patrón sugestivo de tuberculosis miliar, a nivel abdominal corroboró la

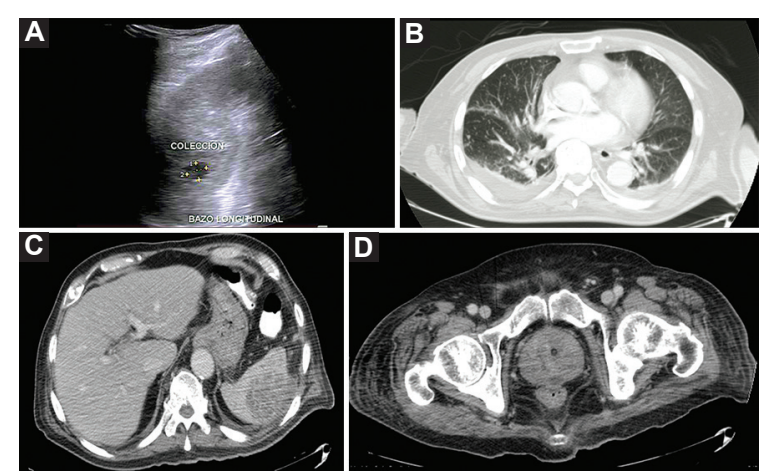

Figura 1. Estudios de gabinete. A: ultrasonido de abdomen superior con presencia de colección esplénica. Parénquima esplénico heterogéneo por la presencia de una imagen hipoecoica, de bordes regulares y definidos, localizada en el polo superior, con dimensiones de $11.6 \times 9.8 \mathrm{~mm}$, avascular en modo Doppler color. B: tomografía computada de tórax contrastada con evidencia de múltiples nódulos centrolobulillares pulmonares de distribución difusa que miden entre 2 y $4 \mathrm{~mm}$, así como áreas de vidrio despulido y engrosamiento bilateral de predominio basal. Espacios pleurales con ocupación de material hipodenso en su interior, de predominio derecho, con unidades de atenuación de hasta $13 \mathrm{UH}$ sin reforzamiento al medio de contraste, que condiciona atelectasia basal pasiva. C: tomografía computada de abdomen contrastada que evidencia el bazo aumentado de tamaño, con dimensiones de $108 \times 52 \times 115 \mathrm{~mm}$ y volumen de $337 \mathrm{ml}$, parénquima heterogéneo a expensas de imagen hipodensa con realce periférico al medio de contraste de hasta $84 \mathrm{UH}$, de $64 \times 47 \times 53 \mathrm{~mm}$, con un volumen de $83 \mathrm{ml}$. Imagen hipodensa periférica adyacente al bazo que mide $25 \times 10 \times 27 \mathrm{~mm}$. Estriación de la grasa periesplénica. D: tomografía computada de pelvis contrastada que muestra una próstata con dimensiones de $17 \times 30 \times 23 \mathrm{~mm}$ parénquima heterogénea por presencia de colección que mide $14 \times 8 \mathrm{~mm}$ y corpúsculos aéreos en su interior.

colección en bazo y a nivel pélvico evidenció una pequeña colección en la próstata.

En días posteriores el paciente desarrolló inestabilidad hemodinámica, con requerimiento de vasopresor a base de noradrenalina y pobre respuesta a los antibióticos de amplio espectro. Por el estado clínico del paciente, se decidió extender el protocolo de estudio en búsqueda de otros focos infecciosos. Los hemocultivos resultaron sin desarrollo y en el urocultivo creció Candida albicans, por lo que se agregó tratamiento dirigido, sin mejoría clínica.

Ya que el foco infeccioso documentado fue el bazo, se procedió a realizar una esplenectomía con extracción de la pieza quirúrgica completa, con cápsula esplénica íntegra con evidencia de plastrón de epiplón en la cara lateral que cubría la lesión (Fig. 2).

El estudio histopatológico de la pieza quirúrgica reportó una lesión difusa, de bordes irregulares, de $5 \times$ $4 \mathrm{~cm}$, de aspecto caseoso, con evidencia de granuloma en la pulpa blanca y células gigantes multinucleadas de tipo Langhans (Fig. 3). Se realizaron tinción de 


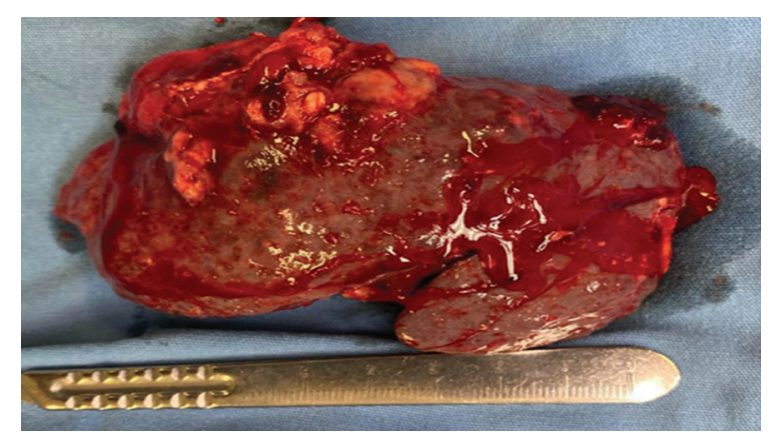

Figura 2. Pieza quirúrgica. Bazo de $9 \times 7 \times 4 \mathrm{~cm}$, con presencia de epiplón en la cara lateral.

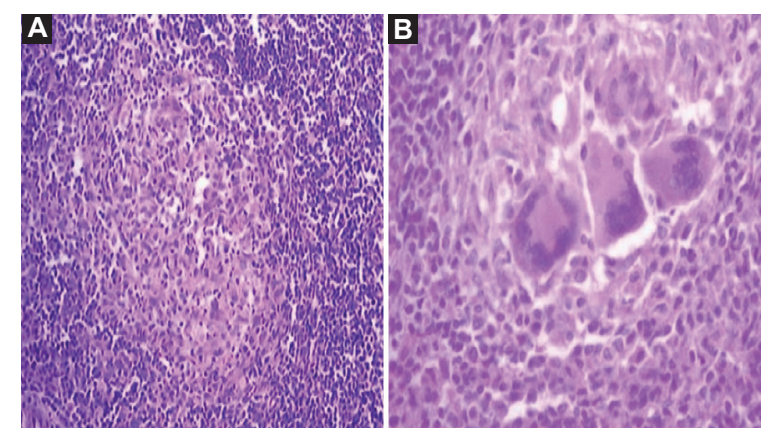

Figura 3. Cortes histológicos de bazo. A: se observa la presencia de granuloma en pulpa blanca. B: a mayor aumento, se evidencia la presencia de células de Langhans.

Ziehl-Neelsen y PAS (Periodic Acid-Schiff) que resultaron positivas para bacilos ácido-alcohol resistentes, PCR en tiempo real para $M$. tuberculosis que también fue positiva, y QuantiFERON ${ }^{\circledR}$ TB Gold-Plus en una muestra sanguínea, igualmente positiva.

Tras la confirmación de la tuberculosis esplénica se inició tratamiento específico con cuatro fármacos en fase intensiva, de acuerdo con las recomendaciones vigentes de la Secretaría de Salud de México, con posterior resolución de los picos febriles, resolución del cuadro de choque y mejoría clínica importante, por lo que se decidió el egreso del paciente.

\section{Discusión}

La tuberculosis es una enfermedad infecciosa granulomatosa causada por M. tuberculosis, y es una de las principales causas de muerte en todo el mundo. A pesar de que en la mayoría de los casos presenta un comportamiento crónico, existen también las formas agresivas y de rápida evolución. La Organización Mundial de la Salud estima que la mortalidad es cercana a 4500 personas al día, con una incidencia de aproximadamente 30,000 casos $^{1}$. En cuanto a la Región de las Américas, solo en el año 2016 se registraron 12,458 defunciones, y de ellas, 2569 correspondían a México ${ }^{2}$. La incidencia ese mismo año en nuestro país fue de 21,184 casos, de los cuales 16,913 fueron tuberculosis pulmonar ${ }^{3}$ y solo 3898 tuberculosis extrapulmonar4.

La mayor incidencia se encuentra en las grandes ciudades y los municipios de mayor número de población, como Tijuana, Ciudad Juárez, Veracruz, Reynosa, Tapachula, Monterrey y Tabasco ${ }^{4}$. Por otra parte, es conocido que los estados de inmunocompromiso, como es el caso de la diabetes mellitus, representan un incremento del riesgo de padecer la enfermedad, registrándose una prevalencia de hasta un $20 \%{ }^{5}$ en los pacientes con esta comorbilidad, lo que conlleva una mayor vulnerabilidad para adquirir las formas de presentación atípicas o extrapulmonares ${ }^{6}$.

Si bien el diagnóstico de tuberculosis pulmonar requiere un alta sospecha clínica dada la presentación de síntomas, en las formas extrapulmonares estas manifestaciones son silentes y de comportamiento atípico, retrasando y dificultando el diagnóstico e inclusive en algunos casos siendo hallazgos incidentales ${ }^{5,6}$.

La tuberculosis esplénica sigue siendo extremadamente rara y un reto diagnóstico, pues la clínica es escasa y poco específica ${ }^{7}$. Generalmente, la forma de diseminación es hematógena, como en este caso, en que el patrón tomográfico torácico era sugestivo de tuberculosis miliar. Las manifestaciones de localización esplénica pueden ser la presencia de abscesos únicos o múltiples. En cuanto a los estudios de imagen, a pesar de no existir un patrón radiológico único o específico, se pueden presentar hipoecoicos ultrasonográficamente e hipodensos tomográficamente, con abscesos múltiples hasta en un $62 \%$ de los casos, focos difusos múltiples en un $25 \%$ y absceso solitario hasta en un $6 \%^{8}$. Por lo tanto, para complementar su estudio es conveniente valernos en la mayoría de los casos de procedimientos invasivos, para poder así aislar la bacteria mediante cultivo o estudios moleculares o histopatológicos. Es importante mencionar que en ocasiones la esplenectomía es una intervención diagnóstica y terapéutica ${ }^{7,9}$.

Si bien el bazo no es una de las principales ubicaciones de tuberculosis extrapulmonar ${ }^{10}$, probablemente la dificultad para acceder a su diagnóstico subestime el porcentaje real de casos. Por ello, es importante documentar este tipo de localizaciones. 
La rotura esplénica secundaria a un absceso tuberculoso es poco frecuente, pero cuando llega a suceder puede manifestar datos clínicos de abdomen agudo; el paciente que presentamos posiblemente tuvo rotura esplénica que fue sellada por el epiplón, por lo que no tuvo sintomatología abdominal ${ }^{10,11}$.

El tratamiento generalmente es el mismo que en las formas pulmonares; sin embargo, la duración varía de acuerdo con el sitio específico ${ }^{12}$. A pesar de que en algunos casos la instalación del tratamiento basta para tener un curso clínico favorable, en las presentaciones atípicas y de evolución rápida el pronóstico puede no ser satisfactorio.

El entendimiento de las formas atípicas y la variedad de presentaciones, teniendo en cuenta la alta sospecha del factor epidemiológico de focos primarios y de su diseminación, aumentará la identificación de casos.

\section{Conclusiones}

La tuberculosis sigue siendo una enfermedad infecciosa altamente prevalente y con alta tasa de mortalidad en todo el mundo. Las formas extrapulmonares, aunque son raras, probablemente sean más frecuentes de lo reportado en la literatura debido a su dificultad diagnóstica. El diagnóstico de absceso esplénico tuberculoso solo por sospecha clínica y radiológica es complejo; no existen patrones específicos, por lo que el abordaje diagnóstico debe ser a través de un análisis sistémico y multidisciplinario, incluyendo toma de biopsia y cultivos.

\section{Agradecimientos}

El presente trabajo no podría haberse realizado sin la colaboración de sus integrantes, por su participación y empeño.

\section{Responsabilidades éticas}

Protección de personas y animales. Los autores declaran que para esta investigación no se han realizado experimentos en seres humanos ni en animales.

Confidencialidad de los datos. Los autores declaran que en este artículo no aparecen datos de pacientes.

Derecho a la privacidad y consentimiento informado. Los autores han obtenido el consentimiento informado de los pacientes y/o sujetos referidos en el artículo. Este documento obra en poder del autor de correspondencia.

\section{Conflicto de intereses}

Los autores declaran no tener conflicto de intereses.

\section{Bibliografía}

1. OMS. Día Mundial de la Tuberculosis. Marzo, 2019. [Acceso 10 junio 2020]. Disponible en: https://www.who.int/es/news-room/events/detail/2019/03/24/default-calendar/world-tb-day-2019

2. OPS. Estrategia fin de la TB: principales indicadores en las Américas. 2019. [Acceso 17 junio 2020]. Disponible en: https://www.paho.org/hq/ index.php?option=com_docman\&view=download\&slug=estrategia-fin-de-la-tb-principales-indicadores-en-las-americas-2019\&Itemi$d=270$ \&lang=pt

3. CENAPRECE. Casos nuevos de tuberculosis pulmonar Estados Unidos Mexicanos 1990-2016. México. [Acceso 17 junio 2020]. Disponible en: http://www.cenaprece.salud.gob.mx/programas/interior/micobacteriosis/ descargas/pdf/6CasosTbOtras16.pdf

4. CENAPRECE. Casos nuevos de tuberculosis otras formas Estados Unidos Mexicanos 1990-2016. México. [Acceso 17 junio 2020]. Disponible en: http://www.cenaprece.salud.gob.mx/programas/interior/micobacteriosis/descargas/pdf/8CasosTbTodas16.pdf

5. Pérez Navarro LM, Fuentes Domínguez F, Morales Romero J, Zenteno Cuevas R. Factores asociados a tuberculosis pulmonar en pacientes con diabetes mellitus de Veracruz, México. Gac Med Mex. 2011;147:219-25.

6. Velásquez MJ, Szigethi QM, Panace VR, Morales IR, Márquez CS, Pefaur PJ, et al. Hepatic-splenic micobacteriosis, unusual form of probable extrapulmonary tuberculosis. Case report and review. Rev Chilena Infectol. 2007;24:59-62.

7. Pratap V, Sinha SP, Bumb SS, Bhaskar DJ. Splenic tuberculosis: a rare entity. BMJ Case Rep. 2014;2014:bcr2013202828.

8. Dixit R, Arya MK, Panjabi M, Gupta A, Paramez AR. Clinical profile of patients having splenic involvement in tuberculosis. Indian $\mathrm{J}$ Tuberc. 2010;57:25-30.

9. Rodarte-Shade M, Díaz-Elizondo JA. Tuberculosis esplénica. Infecciones Quirúrgicas. 2012;13:420-1.

10. Yeo HJ, Lee SY, Ahn E, Kim EJ, Rhu DG, Choi KU, et al. Rotura esplénica espontánea como reacción paradójica durante el tratamiento de la tuberculosis esplénica. Tuberc Respir Dis (Seul). 2013;75:218-21.

11. Safioleas MC, Stamatakos MC, Safioleas CM, Diab Al, Agapitos EB. Coexistencia de ruptura esplénica espontánea y tuberculosis del bazo. Saudi Med J. 2006;27:1588-90.

12. Centers for Disease Control and Prevention. Treatment of tuberculosis. CDC; 2018. [Acceso 10 junio 2020]. Disponible en: https://www.cdc.gov/ tb/esp/topic/treatment/default.htm 